\title{
Passive Thermal Control of the Primordial Inflation Polarization Explorer (PIPER) Flight Electronics
}

\author{
Alexander R. Walts* \\ Cryogenics and Fluids Branch, NASA Goddard Space Flight Center, Greenbelt, MD, 20771, USA
}

\begin{abstract}
The scientific balloon program at NASA offers an exciting and open area of opportunity for testing new technologies and for conducting meaningful experimentation at a fraction of the cost of a space mission. This paper outlines a simple thermal model developed and employed for the Primordial Inflation Polarization ExplorER (PIPER). The sub-orbital environment that PIPER operates in hosts an interesting mix of atmospheric and space thermal challenges. The work done was to mitigate thermal loads and passively cool the payload's exterior mounted electronics at an altitude of $36.6 \mathrm{~km}$. This was done by characterizing the thermal environment and then designing solutions for the heat loads through a combined radiation and conduction passive cooling radiator system thermal model. Despite the simplicity and subsequent limitations of the model, as well as some unexpected payload operational events, the model produced results between $0.31 \%$ and $11.8 \%$ difference between the predicted values and measured average temperatures. From these results, the model was able to successfully provide estimates for the electronics temperatures. Additional flights will be needed to eliminate unknowns encountered in this flight in order to further refine the model for higher accuracy.
\end{abstract}

\section{Nomenclature}

$\begin{array}{ll}A I R & =\text { atmospheric infrared radiation }(W) \\ A l b & =\text { Earth albedo }(W) \\ \alpha & =\text { absorptivity of the studied surface (unitless, range } 0 \text { to } 1) \\ A_{\text {rad }} & =\text { radiating area of the studied surface }\left(\mathrm{m}^{2}\right) \\ C & =\text { thermal conductance }(\mathrm{W} / \mathrm{K}) \\ C_{\text {alb }} & =\text { Earth albedo coefficient (unitless, range } 0 \text { to } 1) \\ D S R & =\text { direct solar radiation }(\mathrm{W}) \\ E I R & =\text { Earth infrared radiation }(\mathrm{W}) \\ \epsilon & =\text { emissivity of studied surface (unitless, range } 0 \text { to } 1) \\ F & =\text { geometric view factor (unitless, range } 0 \text { to } 1) \\ I R_{A} & =\text { blackbody infrared loading from the atmosphere }\left(\mathrm{W} / \mathrm{m}^{2}\right) \\ I R_{E} & =\text { blackbody infrared loading from the Earth }\left(\mathrm{W} / \mathrm{m}^{2}\right) \\ \dot{Q} & =\text { total environmental thermal loading }(\mathrm{W}) \\ S & =\text { solar loading flux constant }=1361 \mathrm{~W} / \mathrm{m}^{2} \\ \sigma & =\text { Stefan-Boltzmann constant }=5.67 \mathrm{e}^{-8} \mathrm{~W} / \mathrm{m}^{2} \mathrm{~K}^{4}\end{array}$

\section{Introduction \& Background}

S Cientific ballooning has been a reliable method of data collection for decades. Scientific balloons today can be Slaunched from a variety of locations around the world and are a much lower cost alternative to orbital spacecraft missions [1]. In addition to the cost savings, one major advantage to balloon experiments is the ability to recover the payload following every flight due to the sub-orbital nature of the flight. At the completion of a balloon mission, the payload is detached from the balloon and free falls until the atmospheric density is thick enough for the pre-deployed parachute to catch and then float the payload down to the planet's surface. This transition typically between $27.5 \mathrm{~km}$ and $30.5 \mathrm{~km}$. When the payload separates from the balloon, a tear is introduced into the balloon to release the helium so that

\footnotetext{
* Research Associate, Observational Cosmology Laboratory, NASA Goddard Space Flight Center, Code 665.0
} 
the balloon returns to the surface for collection. Once the payload has been recovered on the ground, it is processed and reflown. This cycle repeats until enough data has been collected to achieve the mission's scientific objectives.

NASA balloons are constructed of $0.002 \mathrm{~cm}$ thick polyethylene film. Conventional balloons are 'zero-pressure' balloons and as such are open to the atmosphere at the bottom. Helium is used for inflation, and although conventional balloons are typically used for missions ranging from a few hours to a few days, they have the capability to carry a payload up to $3,600 \mathrm{~kg}$ to an altitude as high as $42 \mathrm{~km}$ for a duration of up to two weeks. The balloon program's capabilities have been expanded with the introduction of the Ultra-Long Duration Balloon (ULDB) which can achieve a float time of up to 100 days. Helium capacities of the balloons vary as a function of both the payload weight and the desired float altitude. However, most zero-pressure balloons inflate to approximately 40 million cubic meters of volume at altitude [1]. Overall, scientific ballooning has been a reliable and cost-effective means for space observation with interesting overlaps between the atmospheric and cosmic thermal environments. The challenges of this overlap and how they affect PIPER is discussed in later sections of the article.

\section{The Primordial Inflation Polarization Explorer}

PIPER is a balloon-borne instrument to measure the gravitational wave signature of primordial inflation which is the event theorized to have occurred almost immediately following the Big Bang. This is done by analyzing the inflation event's distinctive imprint on the polarization of the Cosmic Microwave Background (CMB). On board PIPER are several advanced scientific instruments such as cold $(1.5 \mathrm{~K})$ optics, 5,120 bolometric detectors, and rapid polarization modulators. The combined data from the instruments will be used to identify the polarization of the CMB resulting from the sought after primordial signal [2]. PIPER is funded and developed through the NASA-Goddard Space Flight Center Observational Cosmology Laboratories.

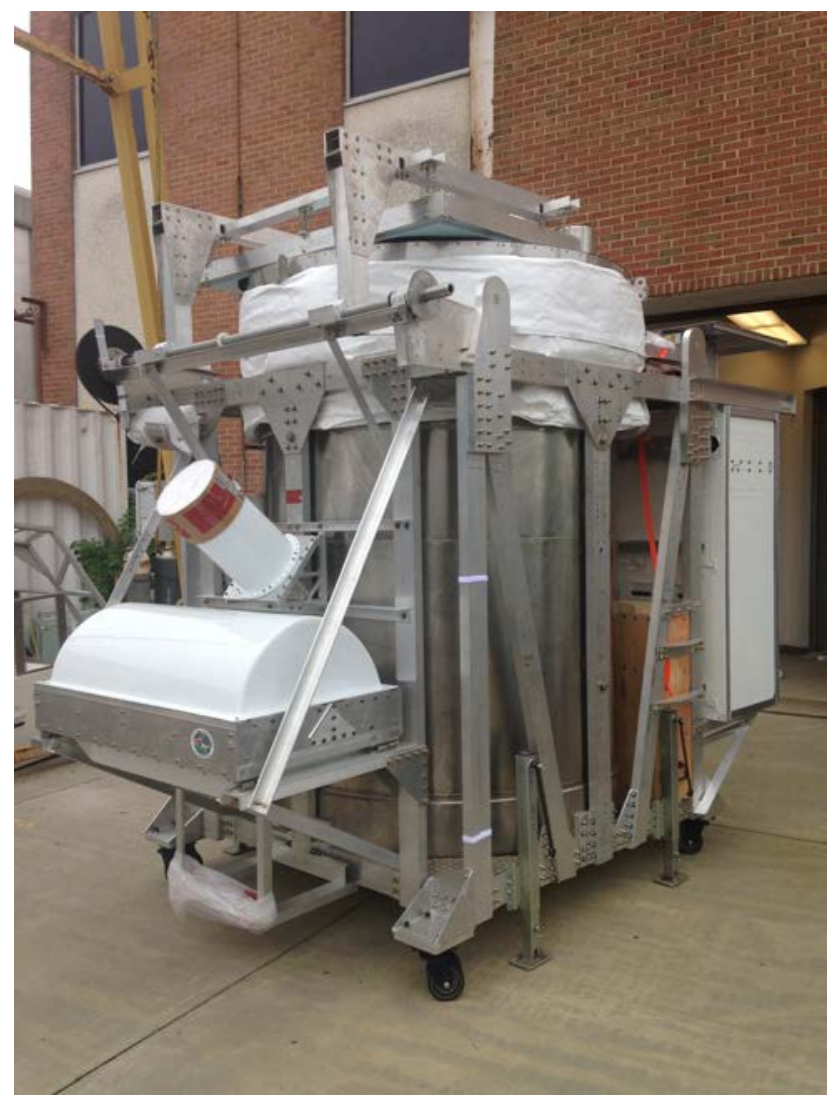

Fig. 1 The PIPER payload.

PIPER will fly several times between the northern and southern hemispheres as there are various parameters that will be measured across a spectrum of frequencies. The total view of all of these flights combined is $85 \%$ of the sky. The 
high sky coverage allows for stronger identification of the desired primordial signals [2]. Currently, PIPER is focusing on flights in the northern hemisphere. These flights are managed by the NASA Columbia Scientific Balloon Facility (CSBF). There are two northern hemisphere flight locations for PIPER. These are in Palestine, Texas and Ft. Sumner, New Mexico. In addition there is a southern hemisphere flight location in Alice Springs, Australia. The choice of the northern hemisphere launch location is based on climatology pertaining to how the upper level winds are predicted to steer the balloon. All flights use a conventional zero-pressure balloon that will lift the PIPER payload to an altitude of $36.6 \mathrm{~km}$. This enables avoidance of almost all atmospheric interference effects in regards to the bolometric detectors.

\section{PIPER Electronics}

In support of the PIPER mission, there are a variety of electronics that govern all aspects of the flight. These include active thermal control of the detector packages, guidance navigation and control, telemetry downlinking, main computers, and power control. Each function is contained within its own electronics box. Each electronics box is housed on subracks inside of an overall container. For PIPER, two overall containers are flown and are 30U in size. This corresponds to $0.50 \mathrm{~m}$ wide, $0.36 \mathrm{~m}$ deep, and $1.33 \mathrm{~m}$ tall. Both $30 \mathrm{U}$ boxes are mounted to the aft side of the payload, one to starboard and one to port. They will be referred to as the starboard and port 30U boxes from this point forward. As mentioned, each $30 \mathrm{U}$ box contains multiple subracks which have varying purposes and internal power dissipations. Table 1 and Table 2 list each subrack, which $30 \mathrm{U}$ box contains it, its purpose, and their estimated power output. Fig. 2 a shows the starboard $30 \mathrm{U}$ box installed on the payload with all of the subracks visible to illustrate the overall layout.

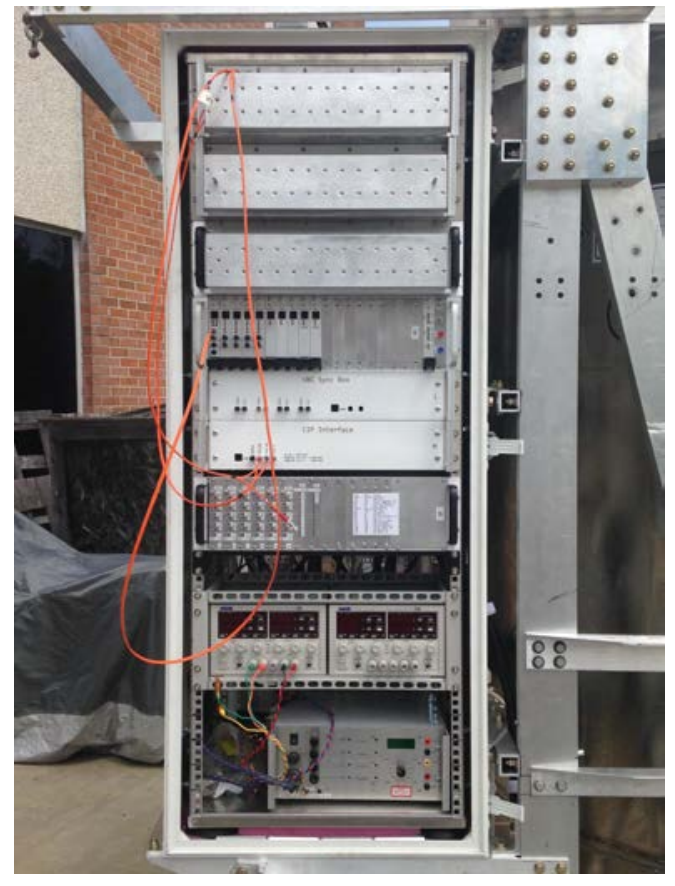

(a) Starboard $30 \mathrm{U}$ box mounted on payload with subracks visible.

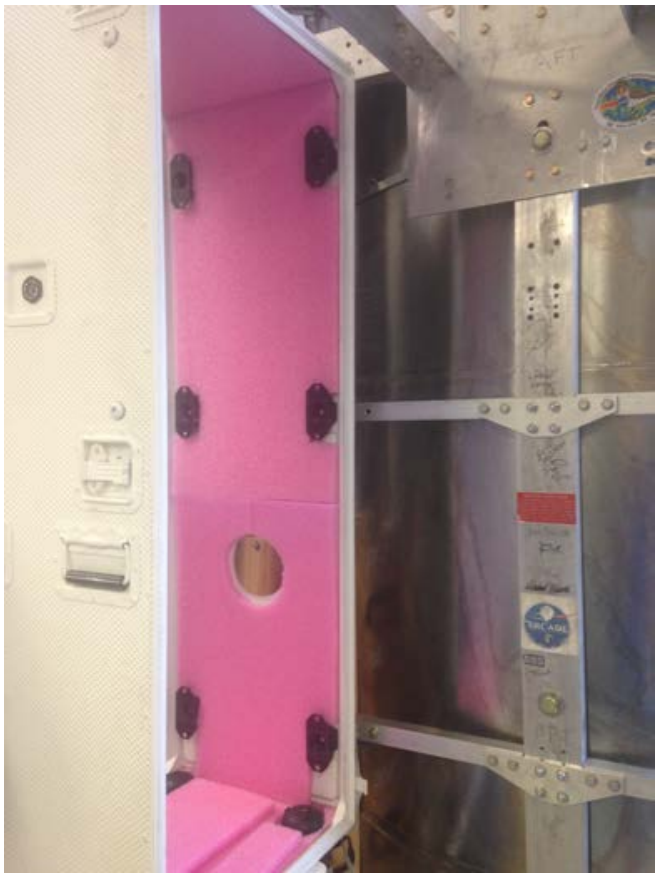

(b) Port 30U empty box with interior foam visible.

Fig. $230 \mathrm{U}$ box layout with and without subrack installation.

Each subrack internal to both $30 \mathrm{U}$ boxes has a low temperature operational limit of $-20^{\circ} \mathrm{C}$ and a high temperature operational limit of $30^{\circ} \mathrm{C}$. Due to time and budget constraints, an active cooling system could not be designed and implemented. As a result, the electronics required the design of a passive cooling system that would hold the electronics to within these temperature limits using the dissipative heat loads shown in Tables 1 and 2 , as well as any associated environmental thermal loads on the cooling system. This entire thermal system needed to be designed, assembled, and implemented with the payload in a period of four months. The optimal operating temperature for the electronics is $0^{\circ} \mathrm{C}$. 
Table 1 Subrack information within the port $30 \mathrm{U}$ box.

\begin{tabular}{|c||c|}
\hline Subrack & Power Output $(W)$ \\
\hline \hline VPM Control & 25 \\
\hline Boost Box & 100 \\
\hline Main Computer & 20 \\
\hline Main Computer & 20 \\
\hline Attitude Control & 10 \\
\hline Power Control & 15 \\
\hline
\end{tabular}

Table 2 Subrack information within the starboard $30 \mathrm{U}$ box.

\begin{tabular}{|c||c|}
\hline Subrack & Power Output $(W)$ \\
\hline \hline Flight Controller & 25 \\
\hline MCE Control & 30 \\
\hline Boost Box & 150 \\
\hline Main Computer & 20 \\
\hline Sink & 10 \\
\hline CIP Interface & 1 \\
\hline Power Control & 20 \\
\hline
\end{tabular}

\section{Model Development}

The sub-orbital environment imposes several different thermal loads on PIPER and its subsequent electronics. The four types of exterior thermal loading are direct solar radiation (DSR), Earth infrared radiation (EIR), atmospheric infrared radiation (AIR) and Earth albedo (Alb). DSR occurs during daytime flights when the PIPER payload is in direct view of the sun. It can be characterized using Eq. 1 1 and is measured in Watts [3]. When analyzing the radiation imparted on the payload as a whole from the Sun, each affected surface must be analyzed individually, and then the sum taken of all the results. The location of the $30 \mathrm{U}$ boxes on the payload is the most important factor in determining the resulting environmental loads. Fig. 3 shows the environment thermal loading on the payload where panel (a) shows daytime loading looking at the aft side of the payload and panel (b) shows daytime loading looking at the top side of the payload. Note that for a night flight, these diagrams are similar, but without direct solar contributions.

As part of the engineering design process, the decision was made to cover all of the interior surfaces of each $30 \mathrm{U}$ box with insulating foam to radiatively decouple those surfaces from the thermal system and reduce the overall heat transfer mechanisms inside each box. Referring to Fig. 3, the interior bottom of each $30 \mathrm{U}$ box was covered with foam to minimize the effects of direct EIR radiation. The surface of each 30U box facing outward was replaced with a designated radiator panel which will be discussed later. The interior surface of each box facing inward was covered with foam to prevent the $30 \mathrm{U}$ box to $30 \mathrm{U}$ box radiative transfer (Fig. $2 \mathrm{p}$ ), and the interior top of each $30 \mathrm{U}$ box was covered with foam due to additional hardware and electronics being located just above each box. Lastly the aft surface of each 30U box (the side facing the Sun) was covered externally by a foam Sun shield to manage incoming direct solar radiation. It was assumed that any surface covered with foam was ideally radiatively decoupled, and that each internal subrack could only thermally communicate with neighboring subracks and the outward facing radiator panels. The interior foam was not enough to fully insulate the system from outside thermal loads, but it was enough to assume radiative decoupling for surfaces that don't face the ground or the Sun (i.e. facing other payload surfaces that aren't as warm). Just the bottom surfaces faced the Earth without external shielding, so the model used an assumption of $20 \mathrm{~W}$ for the heat transfer through the foam from this bottom surface for each $30 \mathrm{U}$ box. Fig $2 \mathrm{~b}$ shows some of the foam following installation. 


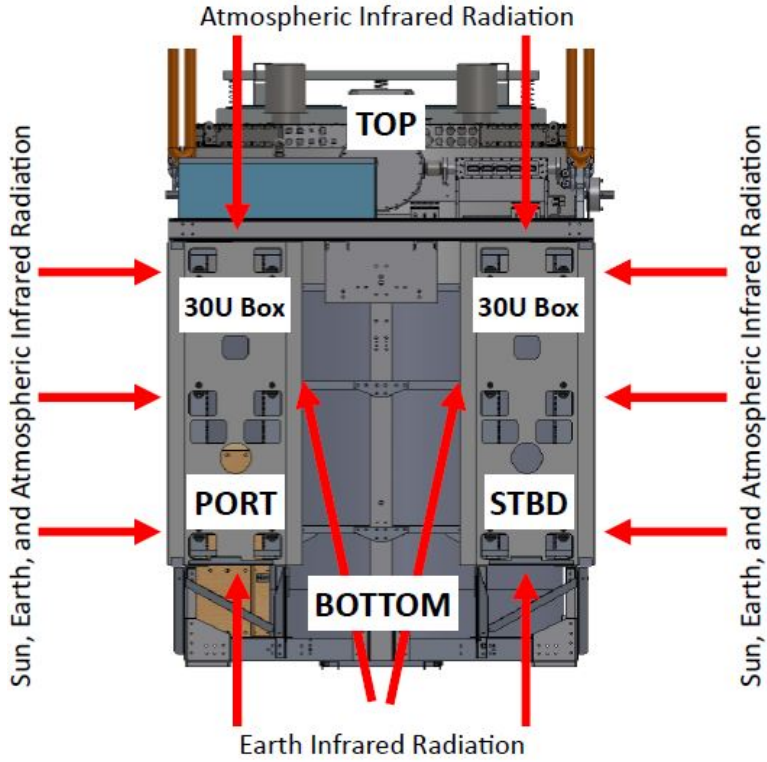

(a) View, vehicle aft

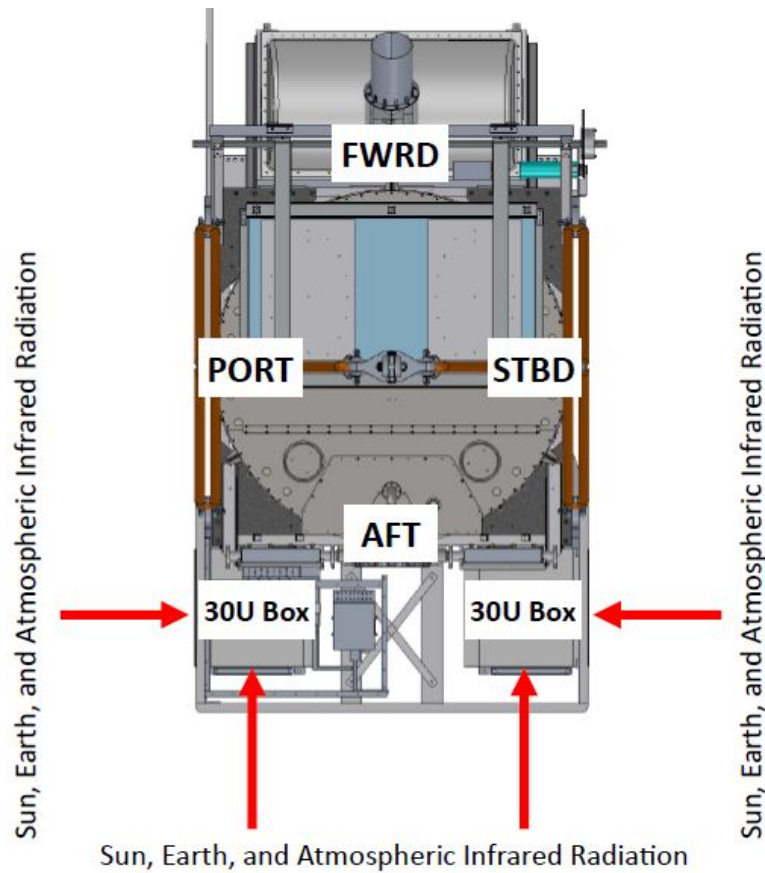

(b) View, vehicle top

Fig. 3 Daytime payload environmental loading on 30U boxes.

\section{A. Environmental Thermal Loading Contributors}

The first type of loading discussed is DSR which is the highest contributing factor by Wattage input to the system. By virtue of flying a daytime mission, this component of thermal loading served as the foundation for understanding the degree of external heating the PIPER electronics would endure. DSR is mathematically defined as follows:

$$
D S R=\alpha A_{\text {rad }} S
$$

EIR is emitted directly from the Earth, and as such it affects the PIPER payload both during the day as well as in the evening. Utilizing the same calculation method for DSR, in order to find the total EIR loading, the load must be found on each individual surface, and then the sum taken of all the results. The equation that governs how much EIR is received by a single surface on the payload is expressed below in Eq. 2] and is measured in Watts [3].

$$
E I R=F \epsilon A_{\text {rad }} I R_{E}
$$

For spacecraft missions, AIR loading is not a factor since significant atmosphere does not exist at orbital altitudes. Although balloon missions achieve very high altitudes, enough atmosphere is still present to where its thermal effects must still be taken into account. The amount of atmosphere present can be seen in Fig. 4 which was produced with the common atmospheric barometric formula. The loading that is received from the atmosphere is still within the infrared spectrum and also measured in Watts. As a result, the equation that governs the loading received by a single surface on the payload is almost identical to Eq. 2 . with the difference being that the emitting body is the atmosphere, not the Earth.

$$
A I R=F \epsilon A_{\text {rad }} I R_{A}
$$



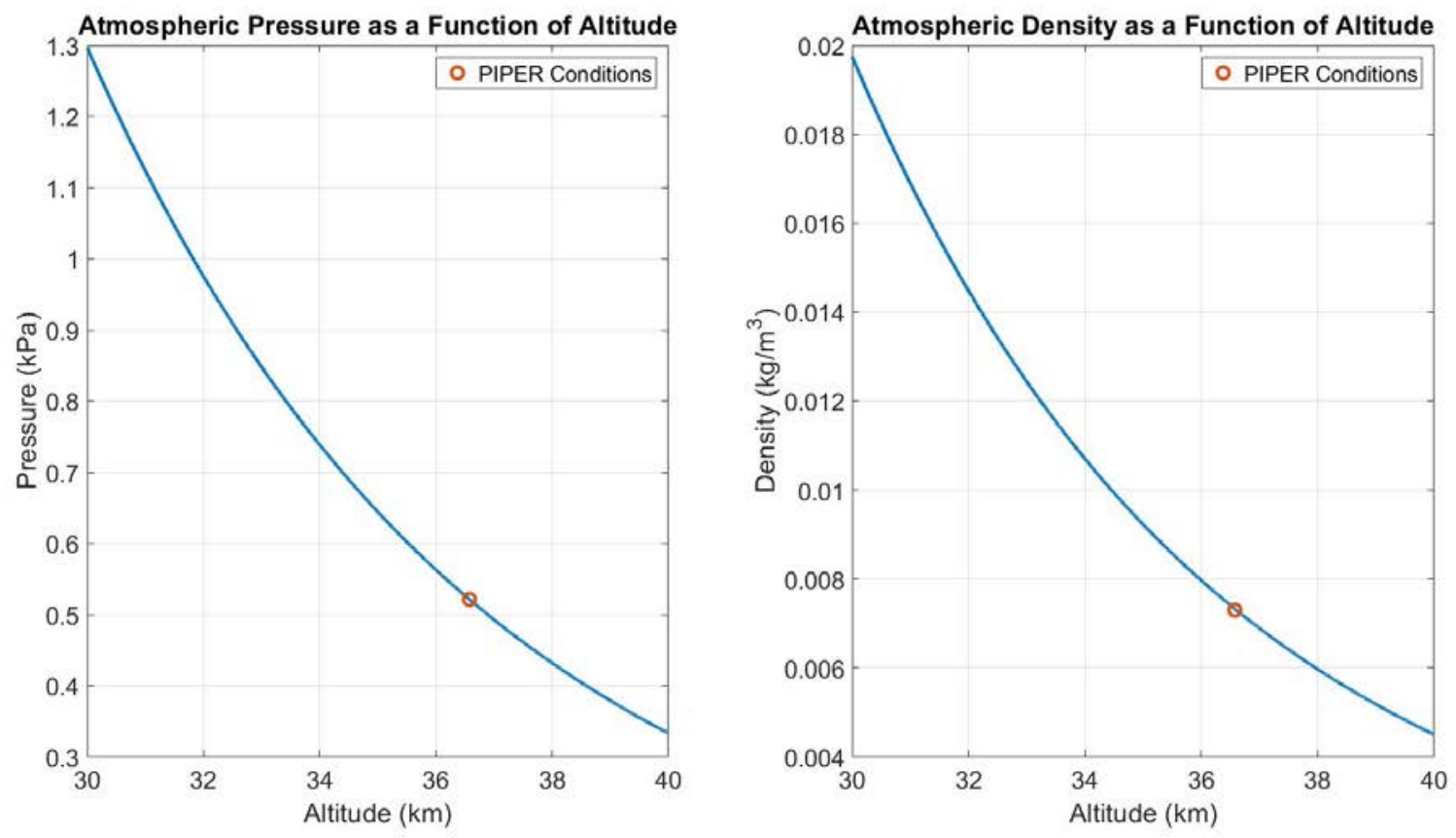

Fig. 4 Standard upper atmospheric properties.

The final source of radiative energy that is analyzed in the sub-orbital environment is Earth albedo. Albedo is a measurement of the Earth's surface reflectivity, and as such, is a measure of how much sunlight is reflected from the ground instead of absorbed by the ground. Since albedo radiation is only possible in the presence of direct sunlight, its effect is not considered for nighttime flights, but is important for daytime flights. The expression for albedo is given in Eq. 4 and like the other expressions, is measured in Watts [3].

$$
A l b=C_{a l b} \alpha A_{\text {rad }} S
$$

With these four different modes of radiative heat transfer, the total thermal loading from the sub-orbital environment onto any surface, including the cooling radiators, of the PIPER payload can be found by summing Eqns. 1, 2,3 , and 4

$$
\dot{Q}=\left(\alpha A_{\text {rad }} S\right)+\left(F \epsilon A_{\text {rad }} I R_{E}\right)+\left(F \epsilon A_{\text {rad }} I R_{A}\right)+\left(C_{\text {alb }} \alpha A_{\text {rad }} S\right)
$$

To solve for $\dot{Q}$ for each side of the two $30 \mathrm{U}$ boxes, the remaining values in each term needs to be determined. Each surface of the 30U boxes was painted in a titanium oxide based white paint such that $\alpha=0.2$ and $\epsilon=0.8$ [4]. This enables each surface to more effectively emit the radiation that is being dissipated which in turn lowers the temperature of the emitting body. Additionally, for sides of the boxes that face the Sun during daytime flights, the low absorptivity value reduces the incoming solar radiation as shown in Eqn. 1 . The values for the surface area are also known for each surface of the $30 \mathrm{U}$ boxes as per the dimensions described in Section III

The only value that remains unknown in equation 5 is the view factor, $\mathrm{F}$, which appears in several of the terms. A view factor is defined as being the fraction of radiation leaving one surface that is intercepted by another surface [3]. The view factor is thus a value that ranges from 0 to 1 and describes geometrically how well one surface can 'see' another surface for the purposes of radiation heat transfer. For example, if there are two actively radiating surfaces facing each other and they are infinitely long and spaced very closely together, the view factor is assumed to be almost equal to 1 . However, if you increase the separation distance between the surfaces, the view factor will decrease since the active surfaces can now see the space surrounding the other surface. For the minimal scenario, if you took two active surfaces and laid them flat on the ground so that they both viewed upward, then the view factor would be 0 . Therefore, one can assume that if you have two active surfaces in a state that is halfway between these extreme conditions (one active surface perpendicular to the other), then the view factor would fall halfway between the given range of values for F of 0 to 1 and would be 0.5 . With this understanding, the view factor of each surface of both $30 \mathrm{U}$ boxes to every source of incoming radiation is now known. 
When applying this principal to the $30 \mathrm{U}$ boxes, one can easily assign the proper values of $\mathrm{F}$ to all the terms in Eqn. 5 . For example, the bottom of each $30 \mathrm{U}$ box looks directly at the Earth and due to the sub-orbital altitude of the mission, the Earth essentially fills the entire field of this surface of view despite the great separation distance. As such, the view factor of the bottom surfaces of the $30 \mathrm{U}$ boxes to the Earth would be equal to 1. Likewise, the top surfaces of the 30U boxes to the atmosphere would be 1 , and each side surface would have a view factor of 0.5 to both the Earth and the atmosphere since these surfaces are normal to the surface of the Earth.

\section{B. EES Finite Element Difference Model}

With all of the internal and external thermal loads on the system now well understood, the effects of these loads on the flight electronics could now be calculated. To perform the calculations, a finite difference model was written utilizing the software, Engineering Equations Solver (EES) utilzing an energy balance approach. Each subrack of each $30 \mathrm{U}$ box was modeled as a single node, the $30 \mathrm{U}$ box skin was modeled as a single node, and each $30 \mathrm{U}$ box radiator was modeled as a single node. The following assumptions were made in regards to the thermal model, and these assumptions were necessary in order to develop a model within the tight four month timeframe of the thermal management project.

- The subracks being stacked have an ideal view of one another (view factor $=1$ )

- No convective effects in the 30U boxes (Fig. 4)

- At steady state conditions, the temperature of all surfaces of each 30U box are isothermal

- At steady state conditions, the radiator panel is isothermal

- The subrack surfaces able to radiate to the $30 \mathrm{U}$ box radiative surface have an ideal view (view factor $=1$ )

- The subrack surfaces able to radiate to the radiator of each $30 \mathrm{U}$ box have an ideal view (view factor $=1$ )

The assumptions concerning view factors are all valid due to the close proximities of all of the participating surfaces. Due to the very low atmospheric density at flight altitude, convective heat transfer effects can be neglected thus supporting the validity of the second assumption. Assumptions concerning isothermal conditions are the weakest and would be the first assumptions eliminated given more model development time.

With each node defined, it simply became a matter of having the nodes communicate to one another using Eqn. 6 Even with the model's relative simplicity in its number of nodes, the system still required a large set of equations and variables to be solved simultaneously. Eqn. 6 was used extensively to describe the radiative thermal communication between all of the nodes. Note that the view factor term, F, was dropped here since the subrack exterior surfaces were positioned close enough to assume a view factor equal to one.

$$
\dot{Q}_{1,2}=\epsilon_{1} A_{1} \sigma\left(T_{1}-T_{2}\right)^{4}
$$

For example, the energy balance for the Main Computer in the port 30U box utilizing Eqn. 6 is shown directly from the EES code in Fig 5 where $Q_{3 R}$ is the radiative transfer from the Main Computer to the 30U box radiator, $Q_{3 s}$ is the transfer from the Main Computer to the $30 \mathrm{U}$ box skin, $Q_{32}$ is the transfer from the Main Computer to the subrack above it (Boost Box) and $Q_{34}$ is the transfer from the Main Computer to subrack below it (Main Computer 2). Lastly, $Q_{b o x 3}$ represents the energy balance of the subrack where $Q_{b o x 3}$ is the $20 \mathrm{~W}$ of heat the subrack produces which is equal to all of the radiative terms being dissipated out of it. Fig. 5 only shows the equations for the main computer subrack in the port 30U box. $Q_{b o x 3}$ is known from Table 1 , but all other heat $(Q)$ and temperature $(T)$ values are unknowns that are solved.

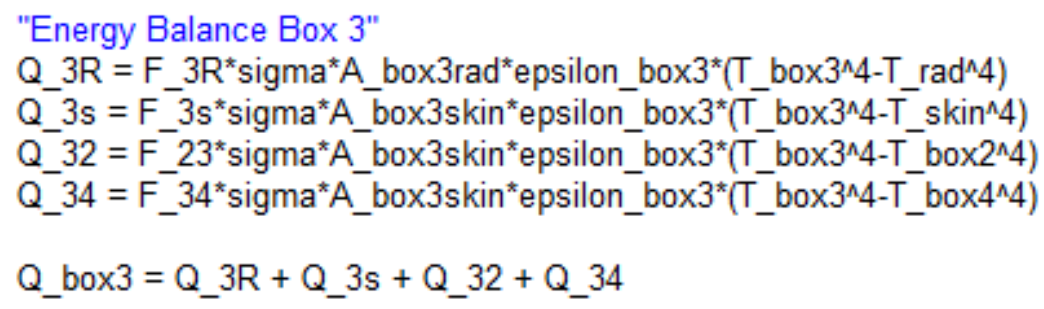

Fig. 5 Port 30U box Main Computer energy balance EES code.

The model also accounted for the introduction of conductive links between the highest power output subracks in each $30 \mathrm{U}$ box to the radiator of that box. Referring to Tables 1 and 2, the Boost Box in the port 30U box and the 
Flight Controller, MCE Control, and Boost Box in the starboard 30U box were all fitted with solid conductive paths to each box's respective radiator. This was done due to the lack of available radiative surfaces on the $30 \mathrm{U}$ boxes and to subsequently ensure that the higher output subracks could effectively dissipate their heat loads with an additional heat transfer mechanism. The equation used in the model to show this conductive link is shown in Eqn. 7 As a result, the model handles both radiative and conductive heat transfer methods simultaneously in the analysis. By taking the $Q_{X R}$ radiation terms from each nodal analysis as well as the conduction terms from any applicable subracks, you get energy balances for the $30 \mathrm{U}$ box radiators and outer total exterior surface area that resemble what's shown in Fig. 6.

$$
\dot{Q}_{1,2}=k\left(\frac{A}{L}\right)\left(T_{1}-T_{2}\right)=C\left(T_{1}-T_{2}\right)
$$

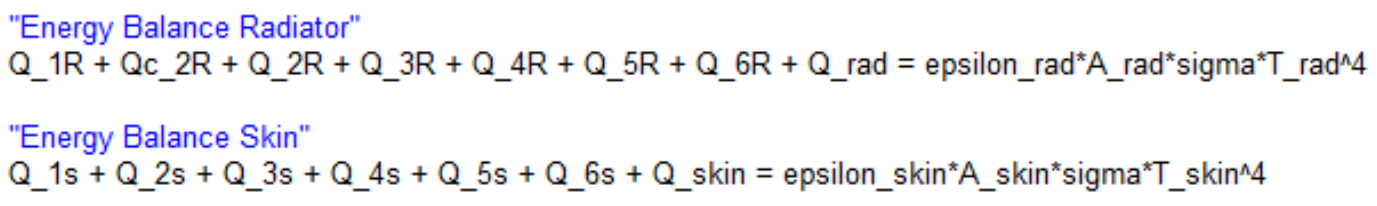

Fig. 6 Energy balances for port $30 \mathrm{U}$ box radiator and box skin.

With Eqns. 6 and 7 the thermal energy balance at each node was established, and a system of equations was solved simultaneously in EES for every $Q$ value in the overall model for each $30 \mathrm{U}$ box and subrack.

\section{Engineering of Thermal Solutions}

The primary method of thermal management for the onboard electronics, as mentioned, was in the form of passive cooling radiators installed on the outer facing surface of each $30 \mathrm{U}$ box as shown in Fig. 7). The thermal model for the electronics was run with the outputs of all the subracks as well as the incoming environmental thermal loads for each $30 \mathrm{U}$ box, and even with the radiators, some subracks were still exceeding their maximum temperature threshold. As a result, and as will be discussed later, thermal shields were also designed, built, and flown on the payload to help block incoming radiation from the Earth and Sun. Instead of limiting the usage and consequent heat output of the electronics, it was decided to reduce the incoming environmental loads. This section discusses all of the engineering design solutions that were implemented to meet the shielding needs of the system.

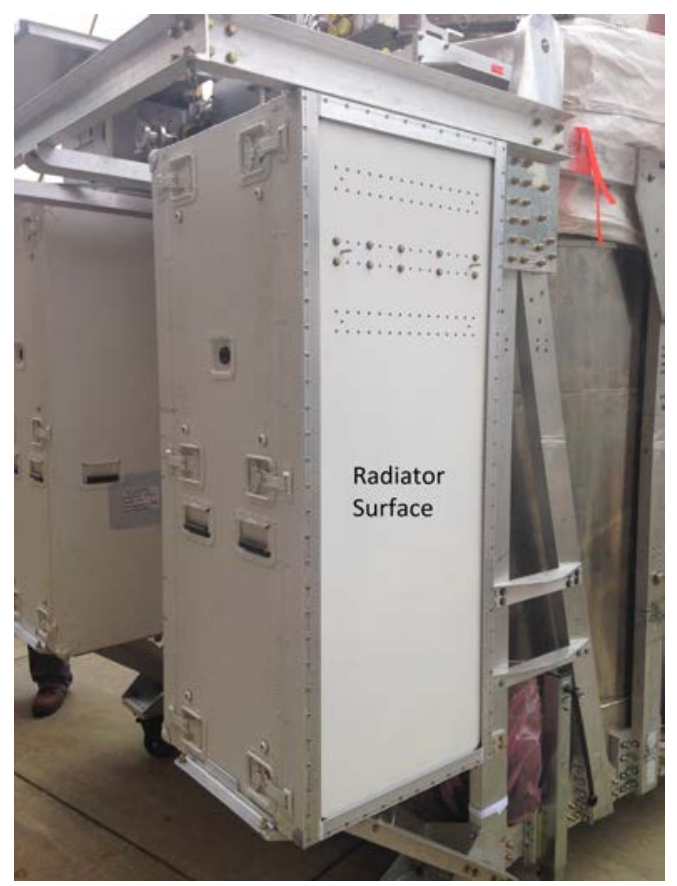

Fig. 7 Starboard $30 \mathrm{U}$ box with radiator panel and conductive bar attachment bolt patterns. 


\section{A. Conductive Heat Transfer Bar Optimization}

As mentioned at the end of the previous section, it was determined that not only would the $30 \mathrm{U}$ box radiators provide passive radiative cooling, but they would also have conductive bars to some of the highest power output subracks in each box. This was determined after preliminary heat transfer calculations showed that through radiative transfer alone, these highest power output subracks would exceed the allowable upper temperature threshold of $30^{\circ} \mathrm{C}$. In designing a conductive path between two points, there are two main critical elements: the area over length ratio $(A / L$ as seen in Eqn. 7), and the mass.

To test both of these elements, a thermal finite element analysis tool was used in SolidWorks CAD software. By Eqn. 7, if you apply a known input heat load, set the sink temperature, and then have the finite element solver produce the warm-end temperature, the $A / L$ ratio can be directly calculated. As a result, an analysis was done on multiple cross-section geometries. The input power and sink temperatures were held constant across all geometries, and the values for input heat load $(100 \mathrm{~W})$ and the sink temperature $(291 \mathrm{~K})$ were chosen to be consistent with the aniticipated values the system would experience during flight. A sample of the results of the analysis are shown in Fig. 8 .

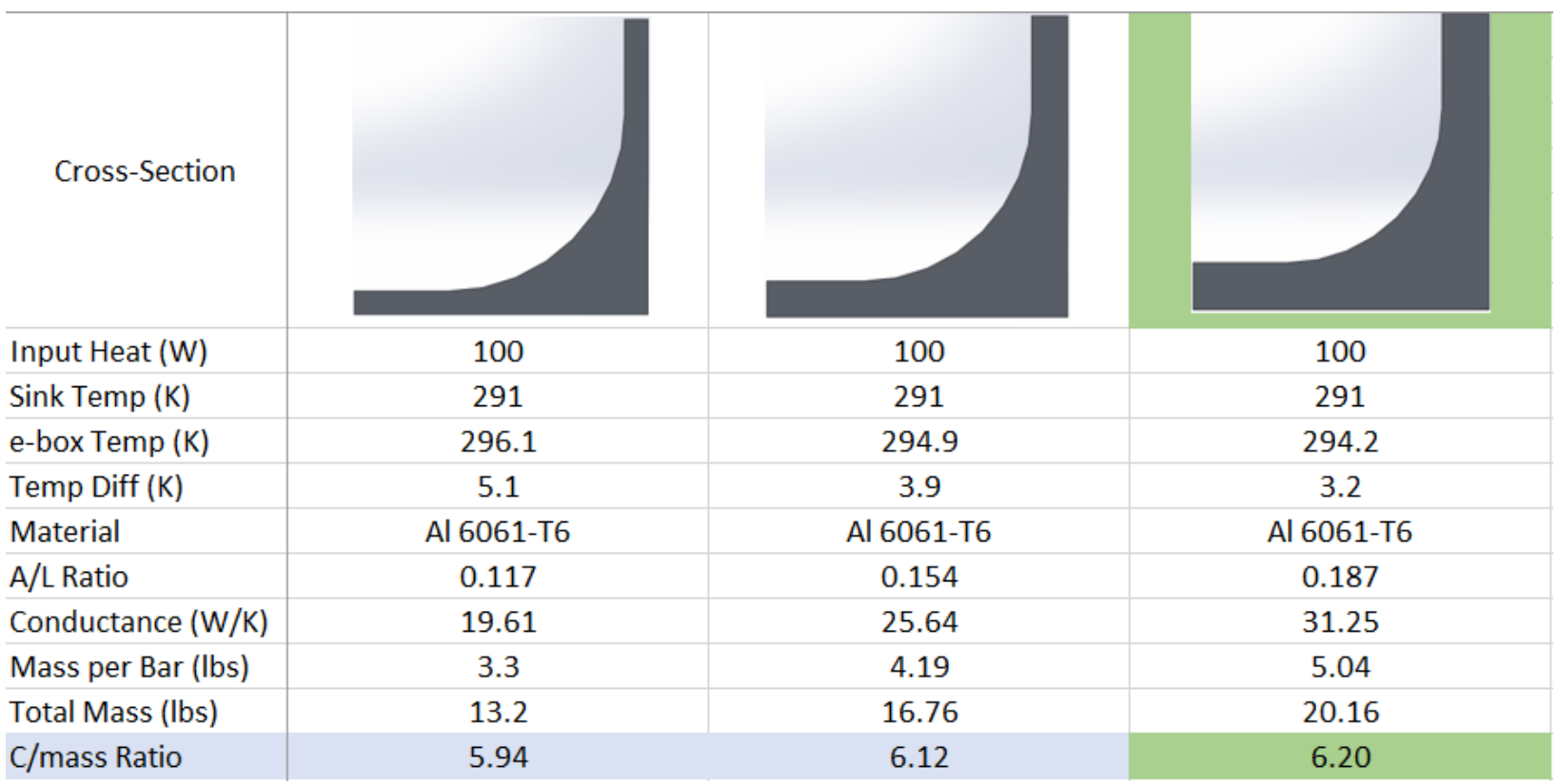

Fig. 8 Conductance bar cross-sections for a few select concepts.

The final design was based on a metric termed by the author as the $C$ /mass ratio which is a measure for how much conductance a certain geometry has per unit mass, thus incorporating both elements most important to the analysis in one metric. The final design choice was based on which cross-section geometry and material produced the highest $C /$ mass ratio while having the combined weight of all four conductive bars equate to approximately 20 lbs due to payload mass limitations. Copper would be an ideal choice for conductivity, but was found to be incredibly overweight. Graphite was also considered, but it showed similar performance to aluminum which is substantially less expensive. In the end, the highlighted green design in Fig. 8 was chosen for its relatively high $C /$ mass ratio at a low weight cost of 5 lbs. per bar. With that cross-section selected, not only was the team confident that the bars had been fully optimized, but the exact $A / L$ ratio could then be integrated with the thermal model to further refine the results.

\section{B. Design and Assembly of Sun Shields}

Since the $30 \mathrm{U}$ boxes containing all of the flight electronics were located on the aft part of the payload, they received direct solar exposure whenever the Sun was above the local horizon. As a result, a large amount of solar radiation would impinge on the boxes and greatly hinder any efforts to keep the boxes cool. To further complicate the problem, in order for the telescope to sweep the sky, the payload would rotate/yaw underneath the balloon $+/-30^{\circ}$ in each direction. As a result, the Sun could radiate on the $30 \mathrm{U}$ box aft sides and radiator panels for any degree of rotation. Using Eqn. 1 , the amount of heat coming into each $30 \mathrm{U}$ box due to direct solar radiation could be characterized as a function of the payload's yaw angle as seen in Fig. 9 It was found with Eqn. 5that without any solar radiation shielding, the 30U 
boxes would be subjected to $542 \mathrm{~W}$ of total environmental loading each, whereas they would only be subjected to $270 \mathrm{~W}$ each if all solar radiation could be mitigated.

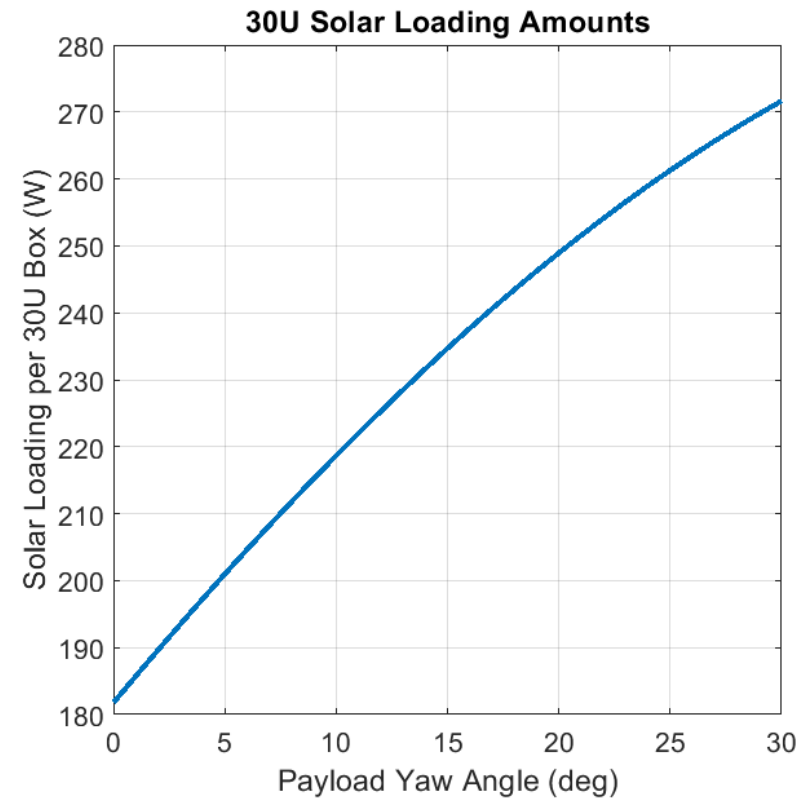

(a) Solar loading characterization due to orientation

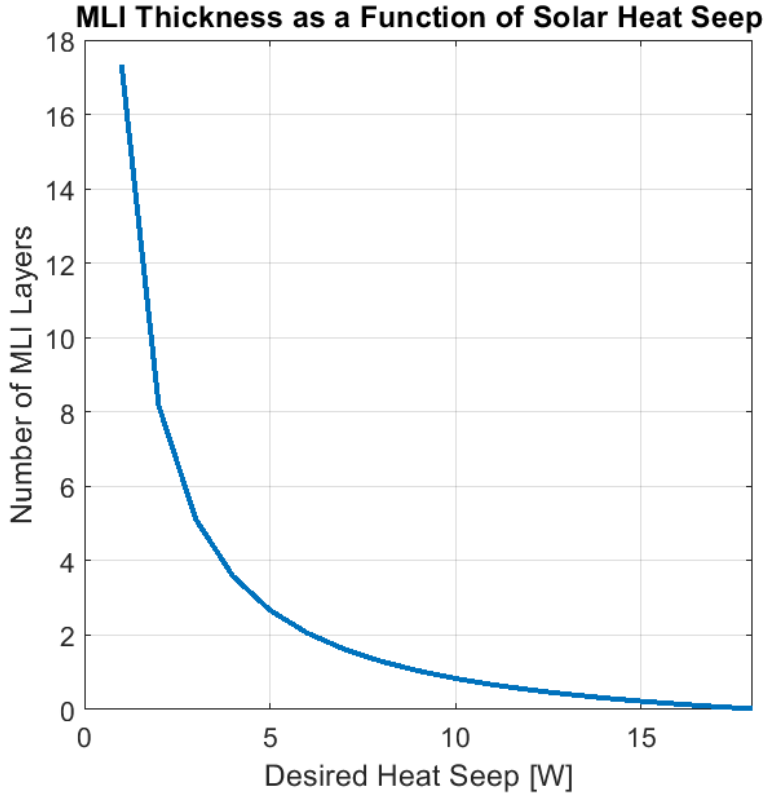

(b) Characterization of number of MLI layers

Fig. 9 Solar shield MATLAB thermal analysis.

The developed solution to this radiation problem was to mount a solar radiation shield to the aft panel of each $30 \mathrm{U}$ box. These 'Sun Shields' would consist of a single panel of insulating foam covered by an MLI blanket. The number of layers in the MLI blanket had to be low enough to permit assembly under a tight time constraint with limited materials while still allowing the blanket to be effective. As a result, from Fig. 9p, it was determined that two layers of MLI with an anticipated $6 \mathrm{~W}$ of thermal energy seep would be acceptable versus $272 \mathrm{~W}$ of unshielded thermal loading based on model predictions. A one layer blanket was considered, however, the heat transfer through the blanket needed to be as low as possible, and a two layer blanket was still easily assembled given project time constraints. This data was calculated by using Eqns. 8 and 9 where $N$ is the number of MLI layers, $\epsilon_{1}$ and $\epsilon_{2}$ are the emissivities of each side of each layer, and $\epsilon^{*}$ is the effective emissivity of the entire MLI blanket. Eqn. 8 solves for the effective emissivity and Eqn. 9 solves for the steady state radiative heat transfer through the blanket [3].

$$
\begin{gathered}
\epsilon^{*}=\frac{1}{(N+1)\left[\frac{1}{\epsilon_{1}}+\frac{1}{\epsilon_{2}}-1\right]} \\
\dot{Q}_{1,2}=\epsilon^{*} A \sigma\left(T_{1}^{4}-T_{2}^{4}\right)
\end{gathered}
$$

With the insulative foam board behind the MLI, it was assumed that by the time the foam board reached a steady state condition where the thermal energy transferred could actually reach the $30 \mathrm{U}$ boxes, the flight would be over. With the full foam and MLI blanket shields installed, the solar radiation contributing to thermal loading was assumed to be negligible. Fig. 10 shows the 30U boxes looking from the aft side of the payload both before and after the application of the assembled Sun shields. Note that the shields extend out on either side of each 30U box so that even when the payload is yawing $+/-30^{\circ}$ in each direction, all surfaces of the boxes are still kept in shadow from the Sun. 


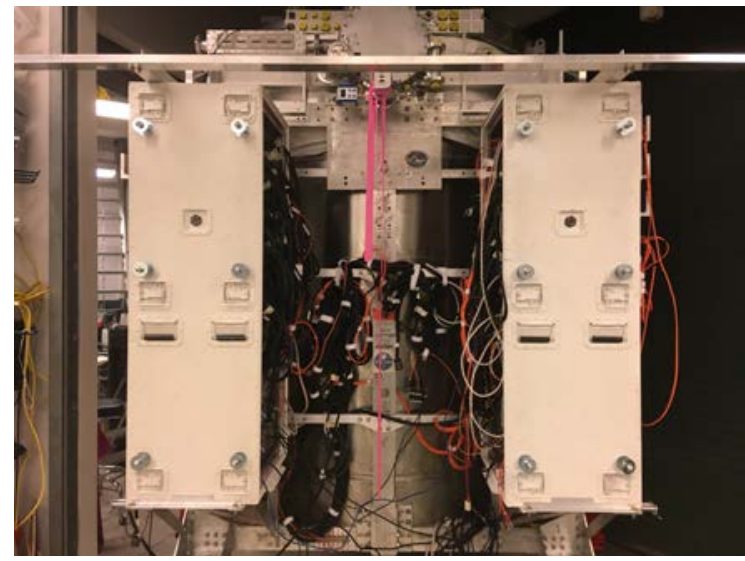

(a) $30 \mathrm{U}$ boxes without Sun shields

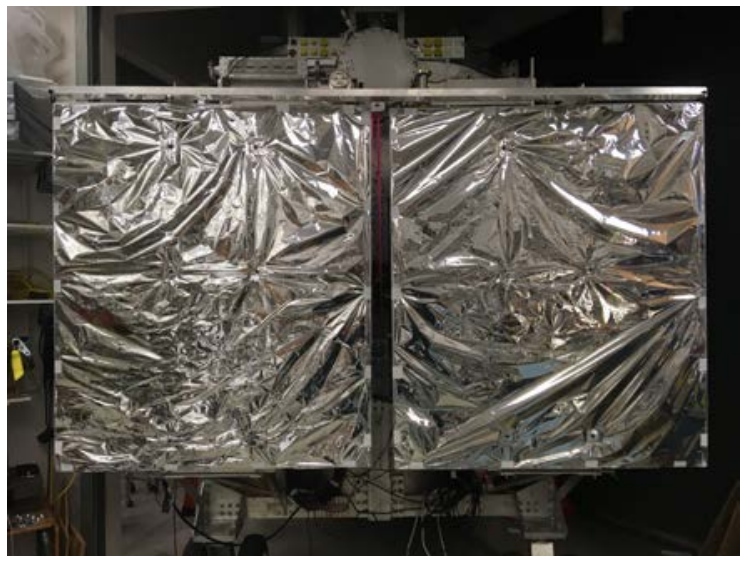

(b) $30 \mathrm{U}$ boxes with Sun shields

Fig. 10 Installation of $30 \mathrm{U}$ box sun shields.

\section{Design and Assembly of Earth Shields}

The final main engineering challenge was finding a way to mitigate the long wave infrared radiation incident on the $30 \mathrm{U}$ electronics boxes from the Earth. Similar to the solar shield concept, the idea here was to block as much of the incoming infrared radiation hitting the $30 \mathrm{U}$ box radiators as possible with 'Earth shields'. These Earth shields were also constructed of two layer Mylar multi-layer insulation blanket (based on the results from Fig. 9p) mounted on insulating foam with the blanket facing Earth. Their orientation was such that they extended out from the bottom of each $30 \mathrm{U}$ box, pointing in the starboard and port directions (depending on the box) and running parallel to the surface of the Earth. Images of the orientation are seen in Fig 11 . Additionally, the tops of the shields had a single layer of Mylar so that the radiators would 'see' the temperature of the ambient atmosphere rather than the temperature of a warming piece of foam.

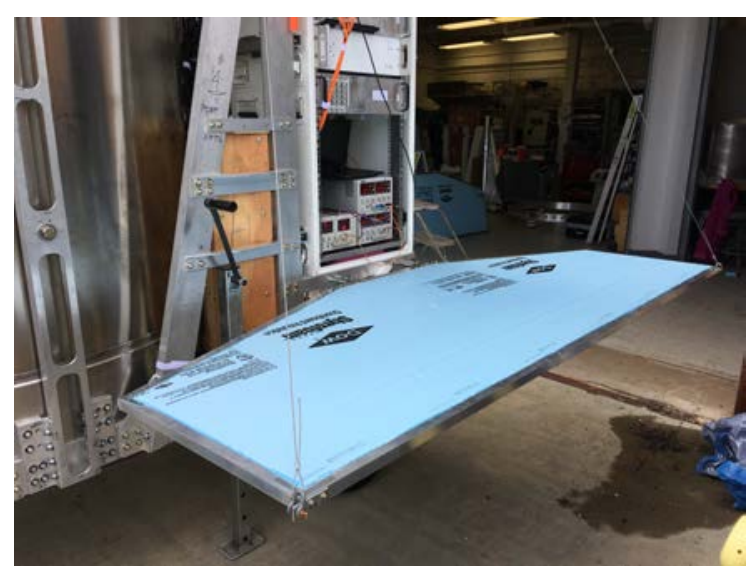

(a) Port Earth shield in deployed position

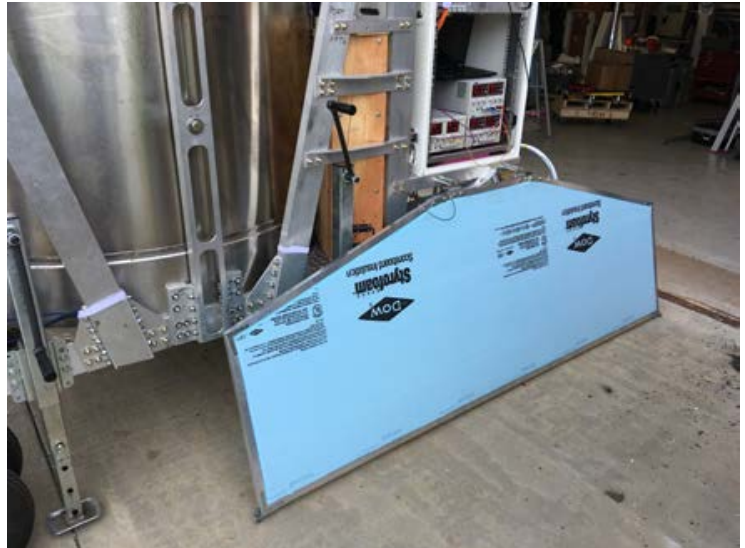

(b) Port Earth shield in stowed position

Fig. 11 Port 30U box Earth shield undergoing fitment check (no MLI installed yet). 
The required geometry for the Earth shields was more difficult to calculate than that of the Sun shields. Compared to the Sun for the Sun shields, the Earth is much larger relative to the payload, and thus completely blocking the view of the Earth from sub-orbital altitudes would have been all but impossible (Fig 13). As a result, it became necessary to determine how far each Earth shield would stick out as well as what angle they would have relative to horizontal (shields angled up towards the radiators block more view of the Earth) while still being a reasonable size. To do this, the view factor of the Earth with respect to the radiator panel was computed using a simple solid view angle calculation. Since the Earth shield would better protect lower sections of the radiator panel than higher sections of the radiator (higher sections could effectively see over the Earth shield to the Earth), the radiator was split into ten equal pieces. The solid view angle analysis was then computed for each piece, and the radiator's total view factor to the Earth was taken as the average of these values for multiple values of Earth shield length and angle. These results are seen in Fig. 12

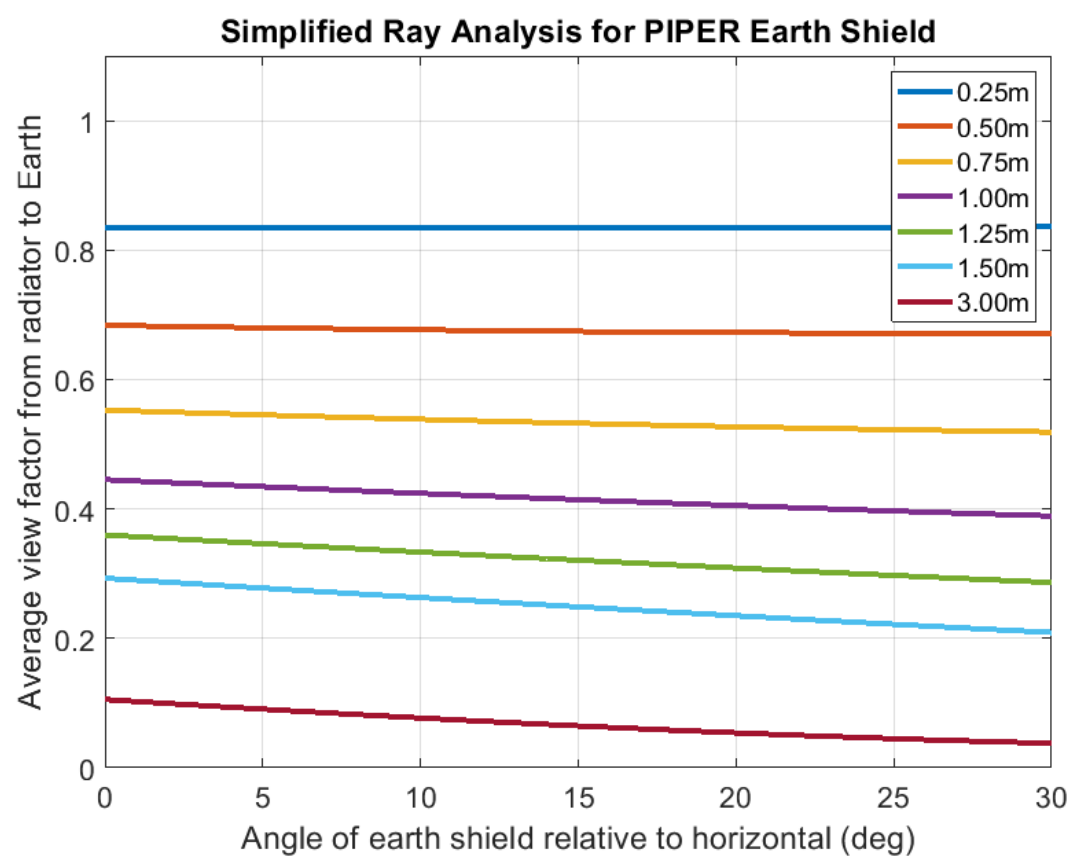

Fig. 12 Radiator's view of Earth as a function of Earth shield extension (in meters) and angle with respect to the radiator.

The angle of the Earth shield relative to horizontal had little effect on the overall view factor except in cases where the Earth shield was very long. One limiting factor on the geometry is that the Earth shields needed to fold out of the way when the payload was on the ground to permit easy access to the $30 \mathrm{U}$ box electronics. This stowed configuration can be seen in Fig. 11p. Due to this constraint, in order for the shields to hang without interfering with the ground, they could only extend out one meter in distance. From Fig. 12, any length shield one meter or less had a view factor that was not significantly dependent upon the Earth shield's angle with respect to the radiator panel. It was also determined that if the Earth shield were angled up towards the radiator panel, that the view factor to Earth went down, but so did the risk of the radiator panels radiating heat onto themselves via a reflective surface angled inwards. From all of this analysis, it was decided that the payload should fly a one meter long shield with zero angle relative to horizontal. Doing so reduced the Earth induced environmental loading on each radiator from $170 \mathrm{~W}$ to $103 \mathrm{~W}$ due to a reduction in the radiator to Earth view factor down to 0.45 . 


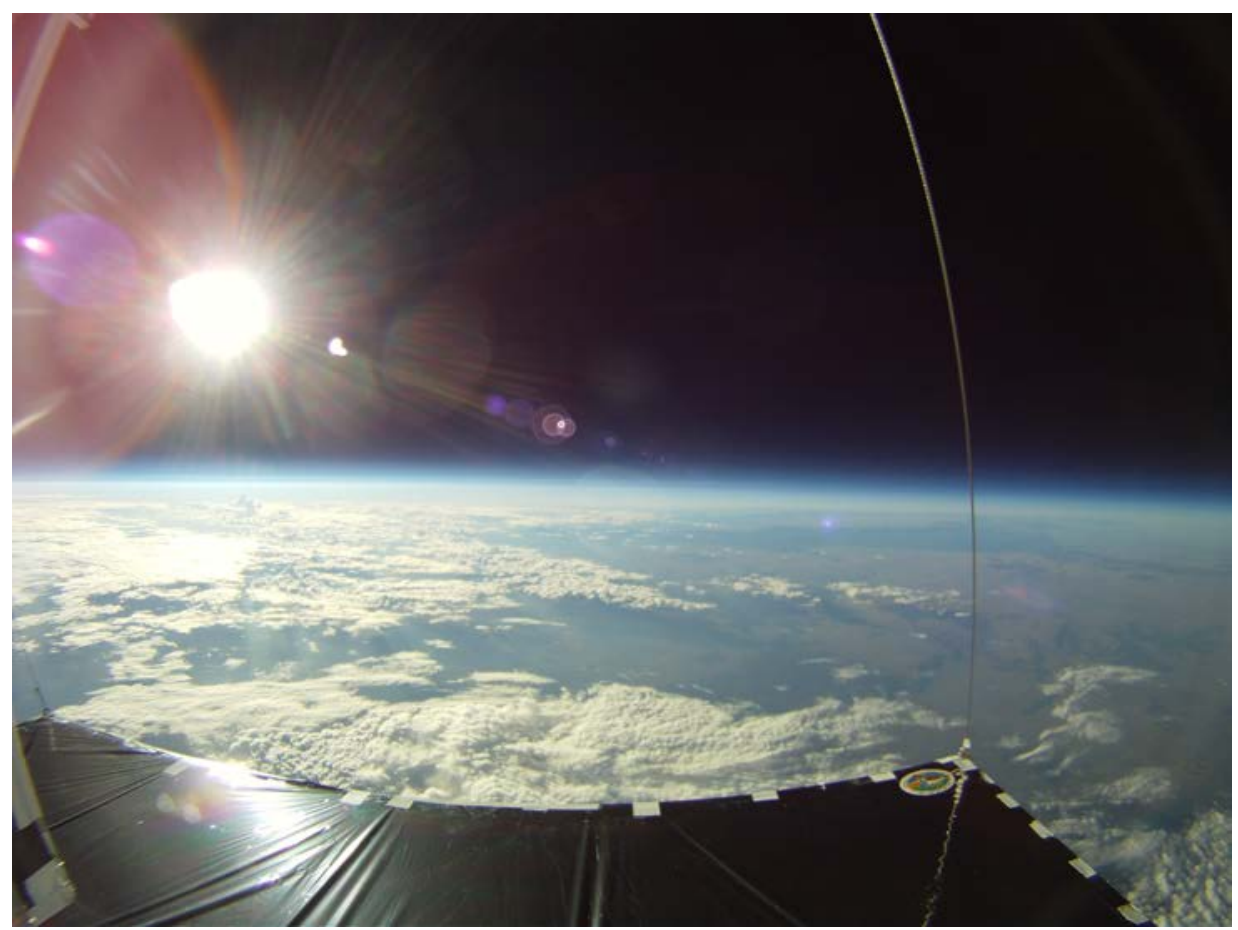

Fig. 13 Earth shield position during flight with relatively large field of view to Earth and relatively small Sun visibility.

\section{Flight Test Results}

Temperature readings were continuously collected from the time of payload launch to the time of payload detachment from the sub-orbital balloon and subsequent descent. Launch occurred on October 13th, 2017 at approximately 9 am MDT, float at altitude was achieved approximately four hours later at $12 \mathrm{pm}$ MDT, and descent at approximately $7 \mathrm{pm}$ MDT. That being said, the data of primary interest is when the mission was floating at the desired mission altitude. It was during this time period that the electronics were fully operational, the payload was experiencing full environmental thermal effects, and mission scientific data collection was occurring. As a result, future plots will show temperature data collected over this time range.

Fig. 14 shows temperature data for the port 30U box radiator with one temperature sensor mounted towards the top of the radiator ('Upper Sensor') and another sensor mounted towards the bottom of the radiator ('Lower Sensor'). Fig. 15 shows the results of the same exact setup for the starboard radiator. These upper and lower sensor positions were chosen since the majority of the electronics, especially the higher power output units, were physically biased towards the tops of the $30 \mathrm{U}$ boxes. As a result, an upper sensor was an obvious choice, with a lower sensor also included to profile the temperature gradient across the radiator panels. Included in the plots are the average measured temperatures for the upper and lower sensors, as well as the overall temperature predicted by the model which will be discussed later.

Fig. 16 and Fig. 17] show the flight temperature results for various select subracks from each $30 \mathrm{U}$ box. The layout and power outputs of these subracks are identified in Table 1 and Table 2 . The subracks were specifically chosen to include the highest power subrack from each box as well as one subrack above and one subrack below, but not directly adjacent to these highest power subracks. This was done because if the model incorrectly predicted the temperature of one subrack, those erroneous temperatures would directly affect the subracks adjacent to the source of the error due to radiative effects. As a result, with these subrack selections, each could be analyzed individually with an acceptable amount of influence from one another without one's error having an overwhelming affect on the others. The measured temperatures, average temperatures, and model predicted temperatures are all plotted, with a discussion of the results in SectionVII 


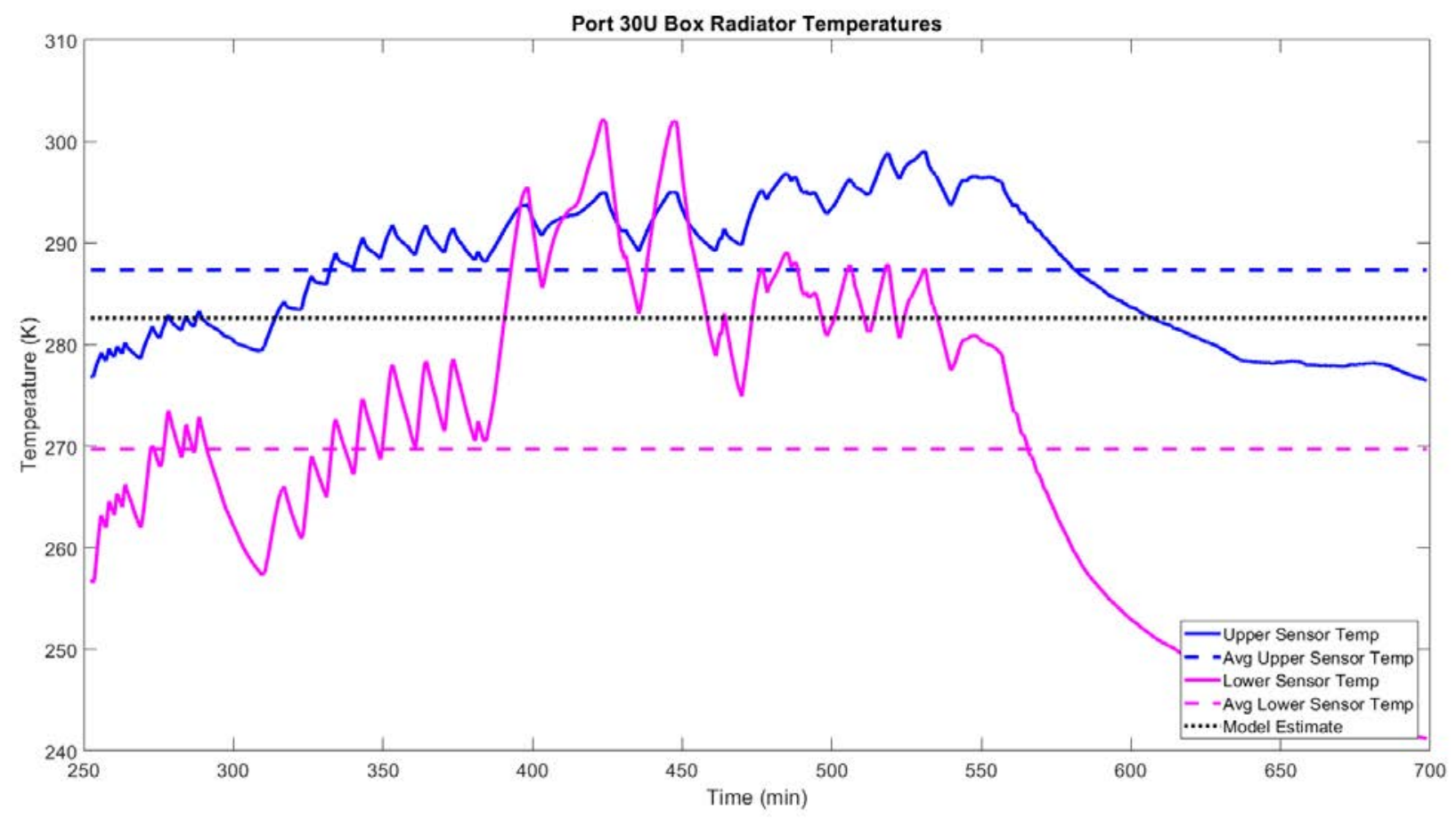

Fig. 14 Model and flight results for the port $30 \mathrm{U}$ box radiator.

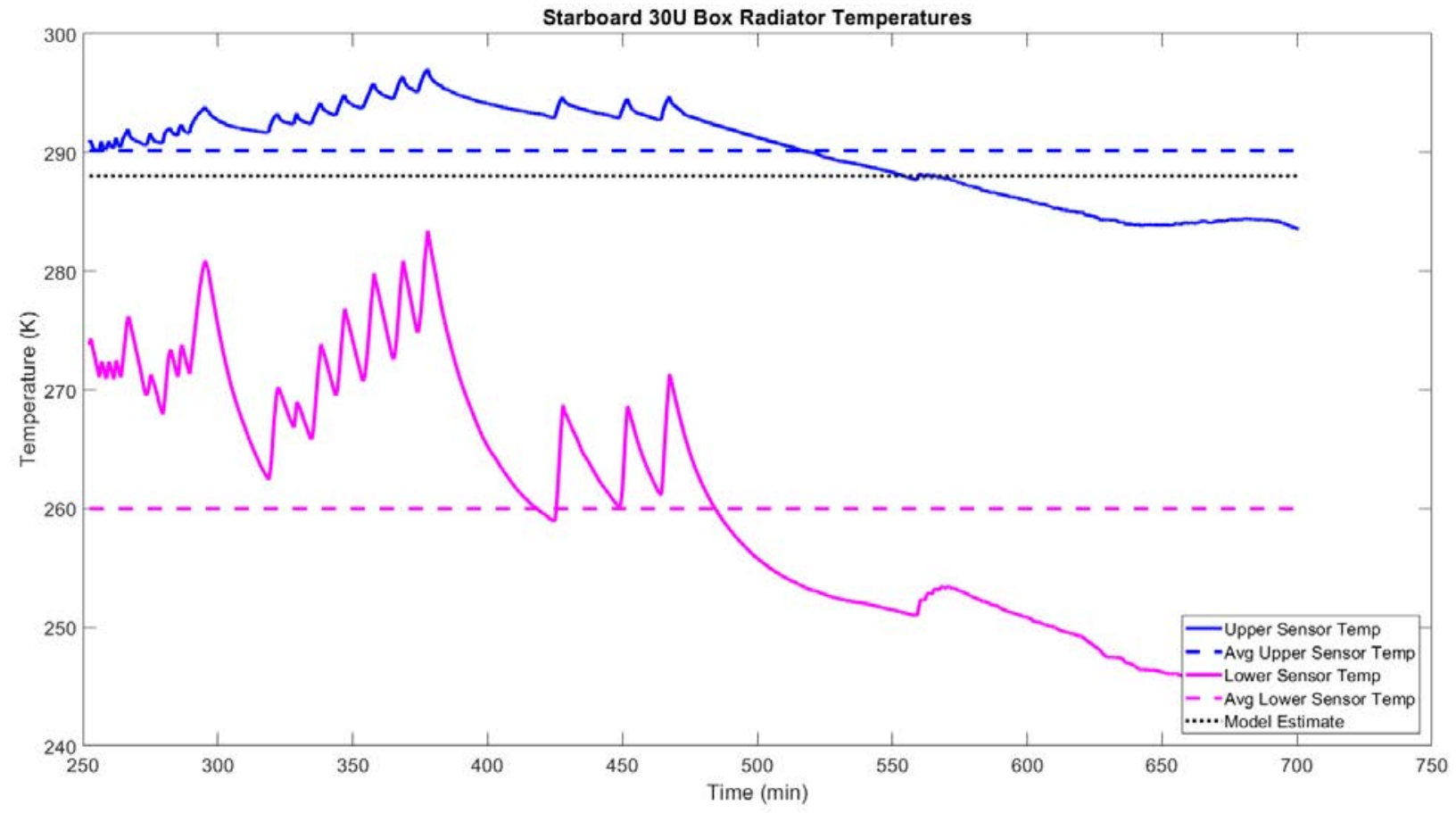

Fig. 15 Model and flight results for the starboard 30U box radiator. 


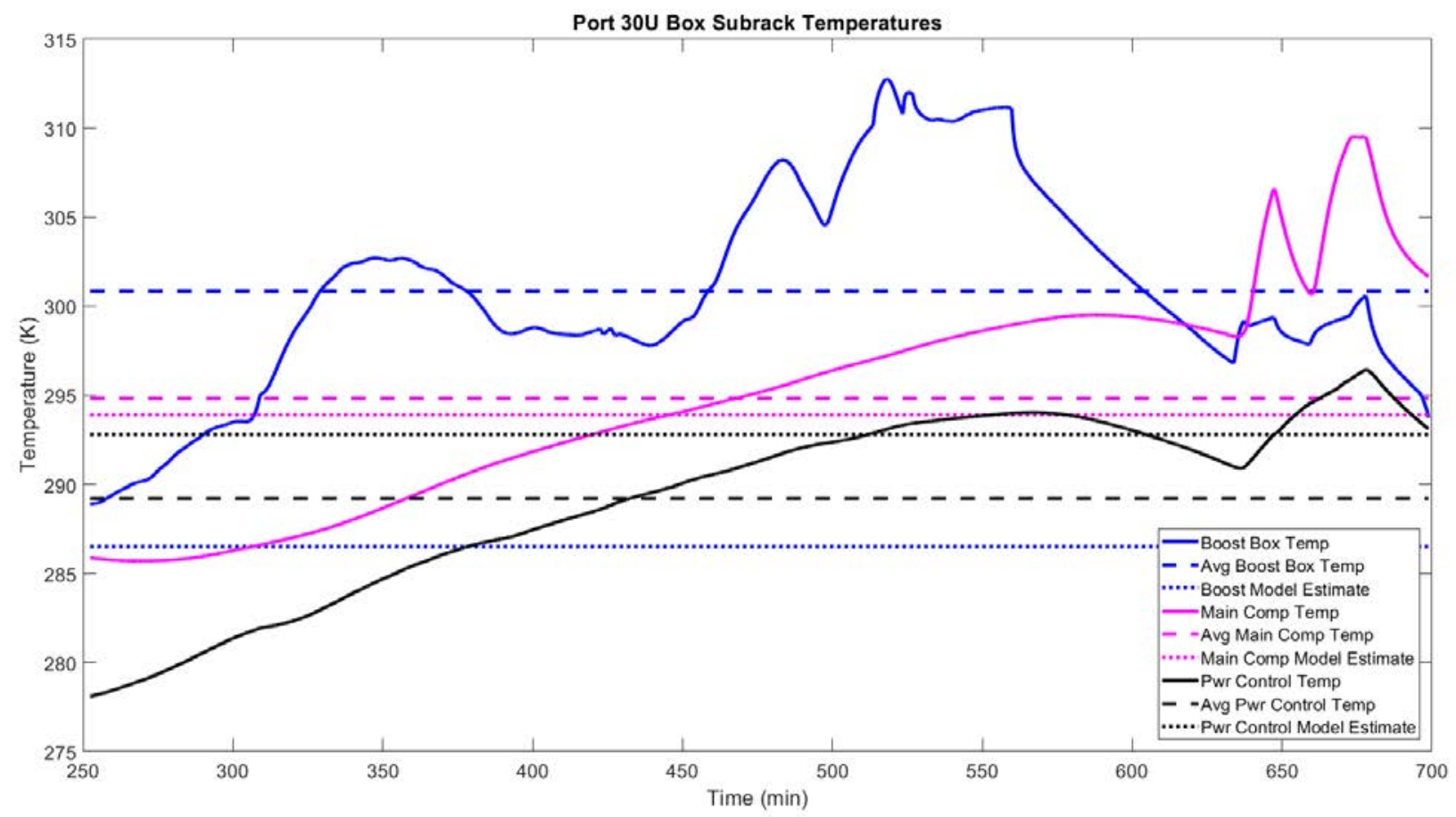

Fig. 16 Model and flight results for select subracks in port 30U box.

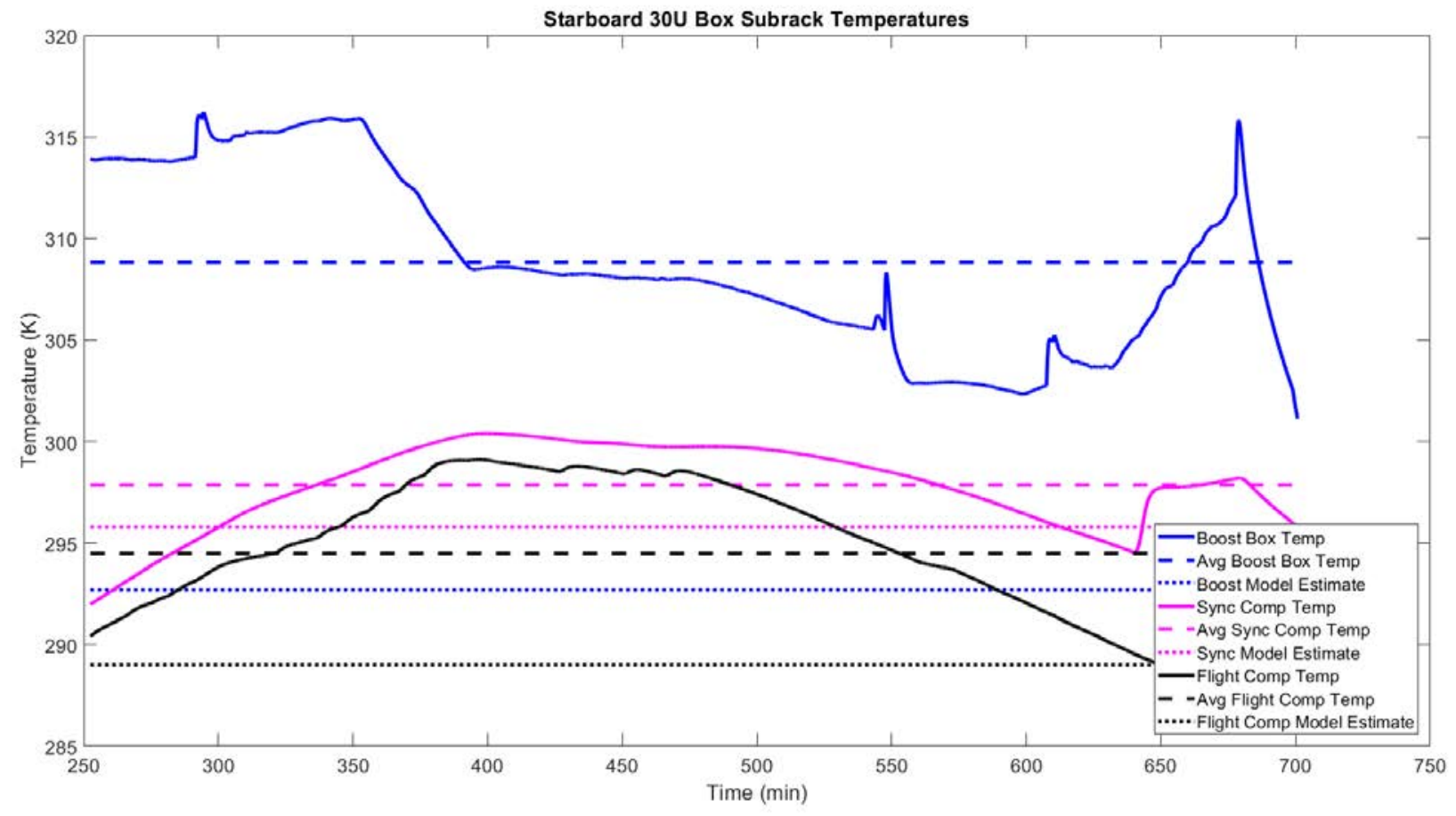

Fig. 17 Model and flight results for select subracks in starboard 30U box. 


\section{Discussion of Results}

The temperature results from the flight are interesting and highlight some shortcomings in some of the model's assumptions. Table 3 provides a listing of the model's temperature predictions, the average measured flight temperature, and the percent difference between them for each component.

Table 3 Summary of flight temperature sensor results.

\begin{tabular}{|c||c||c||c|}
\hline Component & Measured Avg. Temp. $(K)$ & Model Temp. $(K)$ & \% Difference \\
\hline \hline Stbd Radiator Upper & 290.1 & 288 & 0.73 \\
\hline Stbd Radiator Lower & 256 & 288 & 11.8 \\
\hline Stbd Boost Subrack & 308.8 & 292.7 & 5.35 \\
\hline Stbd Sync Subrack & 297.9 & 295.8 & 0.71 \\
\hline Stbd Flight Comp. Subrack & 294.5 & 289 & 1.89 \\
\hline Port Radiator Upper & 287.4 & 282.6 & 1.68 \\
\hline Port Radiator Lower & 269.7 & 282.6 & 4.67 \\
\hline Port Boost Subrack & 300.9 & 286.5 & 4.90 \\
\hline Port Main Comp Subrack & 294.8 & 293.9 & 0.31 \\
\hline Port Power Control Subrack & 289.2 & 292.8 & 1.24 \\
\hline
\end{tabular}

The results show that the model correlated very well to the upper radiator temperature sensors, but not very well to the lower sensors. All of the subracks in each 30U box were stacked from the top down, so the majority of the subracks were in the upper half of each box. As a result, this shows that the model performs well for predicting temperatures at the upper half of the radiator where the majority of radiator to subrack heat transfer interactions were taking place. Due to the large measured temperature difference between the upper and lower radiator sensors, however, it must be concluded that the model's assumption of an isothermal radiator is not valid. Going forward, thermal gradients in the radiators should be accounted for in future missions that use similar components and layouts.

Other areas of good correlation between the model and measured results are in the individual subracks, not including the boost boxes. For all four of the other subracks in Table 3, the percent difference did not exceed two percent. As a result, it can be concluded that the model performs very well for subracks that are not outputting relatively high amounts of power and that rely solely on radiative heat exchange (i.e. no conductive links as with the boost boxes).

For the boost boxes, the percent difference was relatively high when compared to the radiators or other subracks, and there are several explanations for why this occurred. The model takes as an input the estimated power output of each subrack, but for some subracks including the boost boxes, the power output wasn't definitively measured/known until the actual flight. As such, estimates from the electronics specialist were used in the model. As a result, it is certainly possible that the estimates in the model underestimated the actual power output of these subracks. Additionally, as was discussed in Section V, the boost boxes incorporated a direct conductive bar coupling to the radiator to increase the total conductance of heat as necessitated by their dramatically higher power outputs. Along this conductive path, there are areas where thermal conductivity is compromised, such as the interfaces across bolted surfaces. For example, where the conductive bars fasten to the boost boxes and to the radiators, there are a grid of fasteners that apply an even compressive force. The efficiency of the heat transfer across joints such as this are a function of the compressive force, the uniformity of the compressive force, and whether or not a thermal paste is used inside the interface, as well as how much is used. Using SolidWorks FEA, a bolt pattern was designed to try and create as uniform a force as possible. With that in mind, however, the radiators needed to be easily removed in the event of subrack servicing so there was an upper limit to the number of fasteners that could be used. Thermal paste was also used in the interfaces to try and mitigate thermal resistance at the interfaces. Due to the project's extremely tight time limitations, these interfaces could not be characterized fully in the lab, and thus the model operated under the assumption of ideal conduction along the heat flow path. As a result, it is not surprising to see that actual temperatures ran higher than model predictions. The higher than expected temperatures could be attributed to high thermal resistances in these joints. A correction factor for these could be calculated, but it would be prudent to first fully characterize these unknown factors.

Lastly, one more factor that could have contributed to model differences from actual results is in the pointing of the payload itself. Since this maiden flight of PIPER was an engineering test flight, several other subsystems were being tested extensively, and one of these subsystems was the payload's pointing system. During a purely scientific data 
collection flight, the telescope (forward on payload) would never look into the Sun. As a result, the thermal mitigation solutions such as the Sun shields were designed around that constraint. In the case of the engineering flight, the payload pointed in every possible azimuth direction, and actually spun controllably for extended periods of time. Due to this, portions of the $30 \mathrm{U}$ boxes received direct solar exposure that otherwise would not be incident during a non-engineering test flight. The extent of the pointing irregularities are seen in Fig. 18. The upper plot shows the starboard radiator temperatures, and the lower plot shows the Sun azimuth angle in red, and the payload's pointing azimuth in blue. As mentioned, the flight data at full altitude (float) is what was of interest which occurs around time stamp 3 in this figure (this is where the data from Figs. 14 through 17 begin). The blue line shows that at time stamp 4 the payload was already spinning repetitively in full circles. Consequently, each spike in temperatures in the electronics correlates to when the $30 \mathrm{U}$ boxes had direct unplanned for solar exposure. This effect is directly observed in Fig. 13 which shows the radiator panel almost looking right at the Sun. Between time stamps 7 and 8 , the payload begin pointing away from the Sun in the orientation that the solar radiation shields were designed to operate, and thus temperatures steadily dropped. The conclusion from this is that the discrepancies between the model and the actual flight performance can be further attributed to these payload pointing irregularities.

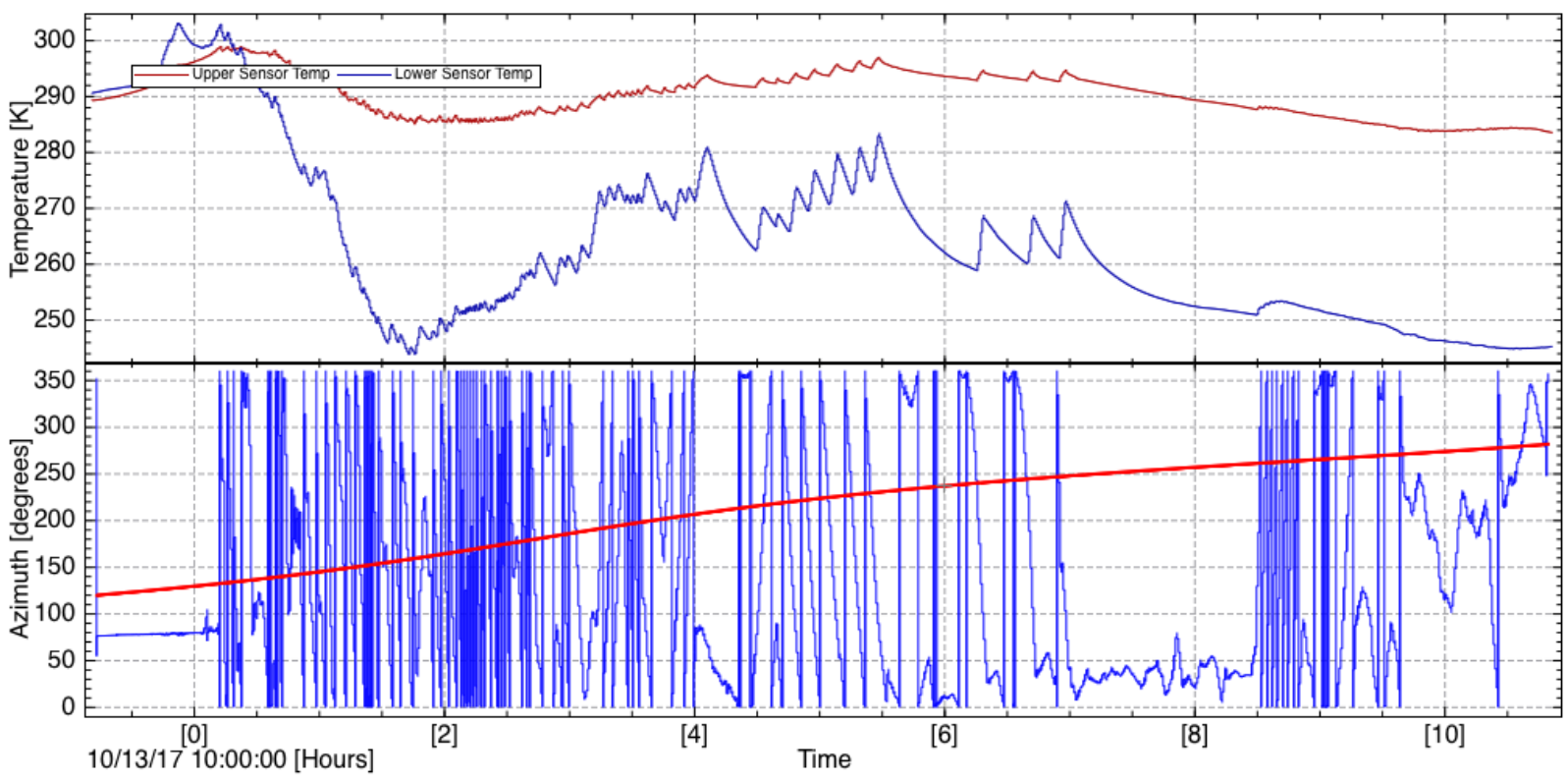

Fig. 18 PIPER and Sun azmiuth angles throughout flight.

\section{Conclusions and Recommendations}

Overall, it is concluded that the model does an adequate job of predicting the exterior mounted electronics temperatures on the PIPER vehicle given the long list of operational and programmatic constraints. The development of a model, design of thermal solutions, and integration of those solutions needed to be completed in the small timeframe of four months with very restrictive mass and financial budgets. Additionally, the variety of unknowns overviewed in the discussion of the flight results introduced a list of means by which error could be introduced. Despite these challenges and uncertainties, the overall temperature requirements for the electronics were met in that the average temperatures of all the subracks remained between the electronics' operational limits of $-20^{\circ} \mathrm{C}$ at the lower end and $30^{\circ} \mathrm{C}$ at the upper end. Looking at Figs. 16 and 17 it is seen that the boost boxes exceeded those temperature limits at times. However, as mentioned, there were several conditions that could have contributed to this, primarily the inconsistencies in the pointing of the payload during testing of the pointing control mechanisms.

Based on the results presented, several recommendations have been identified for consideration before future flights. To start, the thermal resistances of the heat transfer bars at bolted interfaces needs to be more accurately characterized which will eliminate one major unknown. Secondly, the model would be improved by having more accurate knowledge of the power outputs of the individual subracks. The data collected from this engineering flight should act as a starting point for future flights in this regard. Finally, reduced variation in the pointing angle of the payload (as would be 
expected in nominal science flights) would improve agreement between the model and the data. Currently, the model serves as a foundation adequate for successful thermal mitigation in a engineering proof of concept flight, but should have further refinement following the recommendations above before attempting more critical scientific flights.

\section{Acknowledgements}

The author would like to gratefully acknowledge Dr. Alan Kogut, Mr. Paul Mirel, and the rest of the PIPER team. Without their help, guidance, and support, this opportunity and the subsequent work would not have been possible. Additionally, the author would like to thank Dr. Christine Hartzell for her advisement and support, as well as Dr. Eric Silk for his input and assistance.

\section{References}

[1] Johnson, M., “Scientific Balloons [Online],"https://wWw.csbf.nasa.gov/balloons.html 2016. Accessed: 2017-06-26.

[2] Kogut, A., Ade, P. A., Benford, D., Bennett, C. L., Chuss, D. T., Dotson, J. L., Eimer, J. R., Fixsen, D. J., Halpern, M., Hilton, G., et al., "The primordial inflation polarization explorer (PIPER)," SPIE Astronomical Telescopes+ Instrumentation, International Society for Optics and Photonics, 2012, pp. 84521J-84521J.

[3] Silk, E., “ENAE696: Spacecraft Thermal Design,” Lecture notes, University of Maryland, College Park, MD., 2016.

[4] Gilmore, D. G., and Donabedian, M., Spacecraft Thermal Control Handbook: Fundamental Technologies, Vol. 1, AIAA, 2002. 